\title{
Análisis Psicométrico de la Escala Autoeficacia en Conductas de Cuidado de la Salud Física en Universitarios de Primer Ingreso
}

\author{
Martha Ornelas, Humberto Blanco, Judith M. Rodríguez y Francisco J. Flores \\ Universidad Autónoma de Chihuahua. Facultad de Educación Física y Ciencias del Deporte. DES \\ Salud. Calle Escorza 900, CP 31000 Chihuahua, Chih-México. \\ (e-mail: mornelas@uach.mx, hblanco@uach.mx, jurodrig@uach.mx y fflores@uach.mx)
}

Recibido Jun. 06, 2011; Aceptado Jul. 27, 2011; Versión final recibida Ago. 08, 2011

\section{Resumen}

Se estudió las propiedades psicométricas de la escala autoeficacia en conductas de cuidado de la salud física. La muestra fue de 2089 sujetos; 902 mujeres y 1187 hombres, alumnos de primer ingreso a las licenciaturas que se ofrecen en la Universidad Autónoma de Chihuahua en México, con una edad media de 18.23 años. El análisis de las propiedades psicométricas, ha mostrado que una estructura trifactorial es viable y adecuada de acuerdo a los requisitos psicométricos establecidos cuando los informantes son los propios alumnos. La estructura de tres factores, atendiendo a criterios estadísticos y sustantivos, ha mostrado adecuados indicadores de ajuste, de fiabilidad (salvo en los factores Salud Física e Hidratación) y de validez. Además, los análisis factoriales llevados a cabo con las submuestras 1 y 2 comprueban plenamente estos resultados.

\section{Psychometric Analysis of the Self-Efficacy Scale in the Behavior of Physical Health Care on Incoming Students}

\begin{abstract}
Psychometric properties in the self-efficacy scale on behavior of physical health care were studied. The sample was of 2089 individuals: 902 women and 1187 men, incoming students to the majors offered at the Autonomous University of Chihuahua in Mexico, with an average age of 18.23 years. The analysis of psychometric properties has shown that a trifactorial structure is feasible and adequate according to the psychometric requirements established when the informants are the students. The three factors structure, attending statistic and substantial criteria has shown adequate adjustment factors, reliability (except on physical health and hydration factors) and validity indicators. Besides, factorial analyses taken with sub-samples 1 and 2 fully demonstrate these results.
\end{abstract}

Keywords: physical health, scale validity, psychometric analysis, self-efficacy 


\section{INTRODUCCIÓN}

Un ideal común a todas las personas es el de conseguir determinadas metas, la propia motivación impulsa al ser humano a emprender conductas específicas en función de los logros que éste pretende alcanzar. Mas no basta con conocer con claridad aquello que deseamos lograr, ni tampoco el mejor medio para poder conseguirlo. No basta con ser capaz de; es preciso juzgarse capaz de utilizar las capacidades y habilidades personales ante circunstancias muy diversas. La percepción de las personas acerca de su propia eficacia se alza como un requisito fundamental para desarrollar con éxito las acciones conducentes al logro de los objetivos personales. Dicha autopercepción, denominada autoeficacia, ejerce una profunda influencia en la elección de tareas y actividades, en el esfuerzo y perseverancia de las personas cuando se enfrentan a determinados retos e incluso en las reacciones emocionales que experimentan ante situaciones difíciles. En definitiva, las creencias de autoeficacia representan un mecanismo cognitivo que media entre el conocimiento y la acción y que determina, junto con otras variables, el éxito de las propias acciones (Gutiérrez et al., 2011).

Dentro de los contextos educativos ha existido un interés permanente por comprender los factores cognitivos y comportamentales que favorecen o dificultan el desempeño del estudiante en sus labores académicas y cómo éste se relaciona con su desarrollo integral. En el área de la psicología educativa específicamente, el constructo de autoeficacia ha recibido especial atención y se han generado importantes avances de investigación que han contribuido al mejoramiento de prácticas pedagógicas y de enseñanza (Pérez et al., 2011). La investigación empírica ha demostrado ampliamente que la autoeficacia resulta ser más predictiva del rendimiento académico que otras variables cognitivas (Brown et al., 2008; Pérez et al., 2011), también que logra predecir el éxito posterior (Pajares y Schunk, 2001; Pérez et al., 2005) y que es un importante mediador cognitivo de competencia y rendimiento (Valiante, 2000) en cuanto favorece los procesos cognoscitivos.

Por todo ello, en este trabajo que forma parte de un estudio más extenso, cuyo propósito general radica en determinar las diferencias y similitudes entre los estudiantes de primer ingreso a las diferentes licenciaturas de la Universidad Autónoma de Chihuahua en cuanto a su autoeficacia percibida, comprensión lectora y habilidades cognitivas, entre otras; con la finalidad de contar con información valiosa para los sistemas de tutoría y desarrollo personal en nuestra universidad. Se analiza la consistencia interna y la estructura factorial de un instrumento de autoreporte que permite identificar aspectos del cuidado de la salud física, cuyo nivel de autoeficacia percibido por los alumnos de nuevo ingreso representen un área de oportunidad o mejora; en relación al resto de los alumnos, aportando evidencias y datos que propicien la intervención educativa dentro de una perspectiva de atención a la diversidad en el aula. Para contar con un instrumento con las mejores propiedades psicométricas, se realizó el análisis del mismo en dos submuestras, desde la perspectiva del enfoque clásico de la teoría de los test (Muñiz y Fonseca-Pedrero, 2008) y el de los modelos de ecuaciones estructurales (Batista y Coenders, 2000); lo cual permitió realizar estudios paralelos para corroborar y verificar los resultados obtenidos.

\section{AUTOEFICACIA. CONCEPTOS Y APLICACIONES}

La autoeficacia es tradicionalmente entendida como referida a un dominio o una tarea específica. Sin embargo, algunos investigadores también han conceptualizado un sentido general de autoeficacia que se refiere a un sentido amplio y estable de competencia personal sobre cuán efectiva puede ser la persona al afrontar una variedad de situaciones estresantes (Choi, 2004; Luszczynska et al., 2004; Luszczynska et al., 2005).

Para la mayoría de las aplicaciones, Bandura (2001) plantea que la autoeficacia percibida debe ser conceptualizada de manera específica. La autoeficacia percibida hace pues referencia a las creencias de las personas acerca de sus propias capacidades para el logro de determinados resultados. Por consiguiente, el sistema de creencias de eficacia no es un rasgo global sino un grupo de autocreencias ligadas a ámbitos de funcionamiento diferenciado. 


\section{La autoeficacia como constructo}

Bandura considera por tanto a las expectativas de autoeficacia, no como variables globales y estables, sino como cogniciones específicas y cambiantes, que se forman y se reelaboran constantemente a partir del procesamiento y la integración de la información procedente de diferentes orígenes. Es decir, la autoeficacia se refiere a la confianza que tiene una persona de que posee la capacidad para hacer las actividades que trata de hacer; de ahí que los juicios que la persona hace acerca de su autoeficacia son específicos de las tareas y las situaciones en que se involucra, y las personas las utilizan para referirse a algún tipo de meta o tarea a lograr. Por ejemplo Zimmerman (1995) define la autoeficacia referida a la realización de las tareas propias de la escuela como "los juicios personales acerca de las capacidades para organizar y conductas que sirvan para obtener tipos determinados de desempeño escolar". En este sentido, la autoeficacia para la escuela es un concepto muy específico que se refiere a la evaluación que el estudiante hace con respecto a sus capacidades para realizar las actividades propias de la escuela.

Por lo que se puede definir el término autoeficacia como los juicios que cada individuo hace sobre sus capacidades, en base a los cuales organizará y ejecutará sus actos de modo que le permitan alcanzar el rendimiento deseado o como las creencias que tiene la persona sobre sus capacidades para organizar y ejecutar caminos para la acción requeridos en situaciones esperadas o basadas en niveles de rendimiento.

\section{Autoeficacia y cuidado de la salud}

El constructo de autoeficacia ha sido aplicado a conductas tan diversas en distintos dominios de salud como: el manejo de enfermedades crónicas, uso de drogas, actividad sexual, fumar, realizar ejercicio, bajar de peso, y también la habilidad para recuperarse de los problemas de salud o para evitar potenciales riesgos para la salud (Campos y Pérez, 2007). Las investigaciones revelan que altos niveles de autoeficacia tienen consecuencias beneficiosas para el funcionamiento del individuo y su bienestar general (Klein-Hessling et al., 2005).

Sniehotta et al. (2005), investigaron de manera longitudinal a una muestra de 307 pacientes cardíacos quienes fueron motivados a mantener o adoptar la conducta de realizar ejercicios. Estos fueron evaluados inicialmente sobre su intención de realizar ejercicio, luego se realizaron evaluaciones a los dos y cuatro meses siguientes. Los resultados confirmaron que entre los factores evaluados, la autoeficacia funcionaba (junto a las variables de planificación de conducta y control de la acción) como mediadora entre las intenciones iniciales de realizar ejercicio y la actividad física realizada posteriormente.

De acuerdo a las formulaciones de Bandura $(1992,1997)$ cuando un individuo se plantea la posibilidad de llevar a cabo una conducta de salud o cambiar un hábito no saludable hay tres elementos que explican la decisión resultante: 1) la creencia de que una situación determinada es perjudicial; 2) la creencia de que un cambio de comportamiento puede reducir la supuesta amenaza y 3) la creencia de que es suficientemente competente como para adoptar una conducta beneficiosa o para dejar de practicar una que resultaría dañina. Es este último aspecto al que alude el concepto de expectativas de autoeficacia: las creencias acerca de la capacidad para ejercer control sobre la propia conducta y el medio en el que ésta tiene lugar.

Desde la Teoría del Aprendizaje Social de Bandura se asume entonces que las expectativas de autoeficacia son un importante predictor de las intenciones y acciones de los individuos frente a diversas situaciones; ya que un elevado nivel de autoeficacia percibida se ha mostrado como un elemento protector que hace aumentar la motivación, disminuir las alteraciones emocionales, al tiempo que mejora las conductas saludables en el cuidado físico. De hecho, frente a lo difícil que puede ser el motivar a la adopción de conductas que promuevan la salud o el detener conductas nocivas para ésta, la autoeficacia ha mostrado consistentemente ser un factor de gran importancia (Olivari y Urra, 2007). 
La autoeficacia percibida pues, desempeña un papel fundamental en el funcionamiento humano puesto que afecta el comportamiento no sólo de manera directa, sino también por su impacto en otros determinantes claves tales como metas y aspiraciones, expectativas de resultados, tendencias afectivas y percepción de los impedimentos y oportunidades que se presentan en el medio social (Bandura, 1997). Las creencias de autoeficacia influyen en los pensamientos de las personas, en los cursos de acción que ellas eligen para perseguir; los desafíos y metas que ellas se plantean para sí mismas y su compromiso con los mismos; la cantidad de esfuerzo que invierten en determinadas tareas; los resultados que esperan alcanzar por sus esfuerzos; la magnitud de su perseverancia frente a los obstáculos; su resistencia a la adversidad; el nivel de estrés y depresión que experimentan cuando se enfrentan con demandas exigentes del ambiente y los logros que alcanzan.

Con lo dicho hasta aquí se ha querido hacer hincapié en la posibilidad que tienen las creencias de autoeficacia de ser desarrolladas y con ello incrementar la oportunidad de las personas para obtener mejores desempeños. Tratando siempre de afianzar la idea de que darse a la tarea de mejorar la percepción de ser capaz en quien aprende es un objetivo educacional valioso, bajo el supuesto implícito de que su potenciación servirá como vehículo para la mejora de otros resultados tales como el logro académico y la autoestima. Tomando en cuenta que la continua conciencia de fracaso reduce las expectativas de éxito y no favorece en ningún modo ni el aprendizaje ni el desarrollo personal.

\section{METODOLOGÍA}

\section{Sujetos}

La muestra de 2089 sujetos, 902 mujeres y 1187 hombres, todos alumnos de las licenciaturas que se ofrecen en la Universidad Autónoma de Chihuahua; fue aleatoriamente dividida en dos partes utilizando el Statistical Package for the Social Sciences (SPSS) en su versión 15.0 (Tabla 1); con el fin de realizar estudios paralelos que permitieran corroborar y verificar los resultados obtenidos (validación cruzada).

La primera mitad (submuestra 1) quedo constituida por 1065 sujetos; 474 mujeres y 591 hombres. Las edades fluctúan entre los 17 y 20 años, con una media de 18.23 y una desviación estándar de 0.75 años. La segunda mitad (submuestra 2) quedo compuesta por 1024 sujetos; 428 mujeres y 596 hombres. Las edades fluctúan entre los 17 y 20 años, con una media de 18.22 y una desviación estándar de 0.74 años.

Tabla 1: Distribución de los sujetos en la muestra y submuestras

\begin{tabular}{|l|c|c|c|}
\hline & \multicolumn{2}{|c|}{ Submuestra } & \\
\hline \multicolumn{1}{|c|}{ Disciplina } & 1 & 2 & Total \\
\hline Educación Física & 163 & 132 & 295 \\
\hline Ciencias de la Salud & 113 & 116 & 229 \\
\hline Educación y Humanidades & 84 & 84 & 168 \\
\hline Ciencias Sociales y Administrativas & 162 & 138 & 300 \\
\hline Ciencias Políticas & 146 & 143 & 289 \\
\hline Ingeniería y Tecnología & 283 & 309 & 592 \\
\hline Ciencias Agropecuarias & 114 & 102 & 216 \\
\hline Total & 1065 & 1024 & 2089 \\
\hline
\end{tabular}




\section{Instrumento}

Encuesta tipo Likert, asistida por computadora, de 8 ítems relacionados con conductas de cuidado de la salud; donde el encuestado responde, en una escala de 0 a 10, la frecuencia con que actualmente, en forma ideal y si se esfuerza en cambiar, realizaría o manifestaría una acción (Blanco et al., 2007). Se eligió este tipo de encuesta por ser fácil de construir y de aplicar; además, de proporcionar una buena base para una primera ordenación de los individuos en la característica que se mide (Raviolo et al., 2010).

Aún cuando cada sujeto respondió a los 8 ítems del instrumento en tres escenarios distintos: Escenario actual, respondiendo en el contexto: actualmente con que frecuencia realiza determinada conducta o se percibe con determinada característica. Escenario ideal, respondiendo en el contexto: con que frecuencia desearía realizar determinada conducta o percibirse con determinada característica. Escenario de cambio, respondiendo en el contexto: si me esfuerzo en cambiar con que frecuencia realizaría determinada conducta o me percibiría con determinada característica. En el análisis psicométrico solo se utilizaron las respuestas al primer escenario.

\section{Análisis clásico de las propiedades psicométricas de la escala}

Para determinar el número mínimo de factores comunes capaces de reproducir, de un modo satisfactorio, las correlaciones observadas entre los ítems del instrumento (sin considerar los ítems consumo de tabaco y consumo de alcohol), se realizaron sendos análisis factoriales exploratorios con las submuestras 1 y 2, a partir del método de factores principales, tomando como base el criterio de Kaiser-Guttman (Costello y Osborne, 2005), por el que se toman autovalores mayores o igual a la unidad y el análisis gráfico del Scree Test de Cattell (Costello y Osborne, 2005) sobre los autovalores; además de que para garantizar una adecuada representación de las variables (ítems), solo se conservaron aquéllos cuya comunalidad, 0 proporción de su varianza explicada por la solución factorial, fuera superior a .45; después de una rotación varimax (Costello y Osborne, 2005). Luego mediante el coeficiente a de cronbach se estimó la consistencia interna para cada factor retenido como una medida de su fiabilidad (Nunnally, 1991; Elosua y Zumbo, 2008).

\section{Análisis de las propiedades psicométricas de la escala}

Para conducir el análisis factorial confirmatorio en la submuestra 2, se utilizó el software AMOS 16 (Arbuckle, 2007), las varianzas de los términos de error fueron especificados como parámetros libres, en cada variable latente (factor) se fijó uno de los coeficientes estructurales asociados a uno, para que su escala sea igual a la de una de las variables superficiales (ítems). El método de estimación empleado fue el de Máxima Probabilidad; siguiendo la recomendación de Thompson (2004), en el sentido de que cuando se emplea análisis factorial confirmatorio se debe corroborar no sólo el ajuste de un modelo teórico sino que es recomendable comparar los índices de ajuste de varios modelos alternativos para seleccionar el mejor.

Para evaluar el ajuste del modelo se siguieron las recomendaciones antes planteadas, que indican la conveniencia de emplear múltiples indicadores de ajuste. Específicamente, se emplean el estadístico Chi-cuadrado, el índice de bondad de ajuste (GFI), el residuo cuadrático medio (RMSR), el error cuadrático medio de aproximación (RMSEA) y el índice de validación cruzada esperada (ECVI) como medidas absolutas de ajuste. El índice de bondad ajustado (AGFI), el Índice Tucker-Lewis (TLI), el Índice del ajuste normal (NFI) y el índice de bondad de ajuste comparativo (CFI) como medidas de ajuste incremental. El índice de ajuste normado de parsimonia (PNFI), el índice de calidad de ajuste de parsimonia (PGFI), la razón de Chi-cuadrado sobre los grados de libertad (CMIN/GL) y el Criterio de Información de Akaike (AIC) como medidas de ajuste de parsimonia.

Para comprobar la estructura factorial de la escala autoeficacia percibida en conductas de cuidado de la salud obtenida a partir de la submuestra 2 (análisis factorial confirmatorio, primera solución factorial), se realizó un segundo análisis factorial confirmatorio en la submuestra 1. 


\section{RESULTADOS Y DISCUSIÓN}

Análisis factorial exploratorio (primera solución factorial)

Al inspeccionar la matriz de correlaciones entre los 8 ítems de la escala; se observa que 2 de ellos (consumo de tabaco y consumo de alcohol) tienen correlaciones marginales con los otros 6 (Tabla 2); al no considerarlos, el promedio de correlaciones entre los ítems de .218 sube a .335, lo que favorece la posibilidad de realizar un análisis factorial exploratorio. La significación del Test de Barlett (1434.652; $p<.0001)$ y el KMO (.75) mostraron una adecuada correlación entre los ítems y una buena adecuación muestral respectivamente. Detectándose, después de una rotación varimax, una estructura de tres factores: Alimentación, Salud Física e Hidratación. El conjunto de los factores seleccionados explicaron el $75.65 \%$ de la varianza (Tablas 3 y 4 ).

Tabla 2: Correlaciones entre los reactivos de la escala. Submuestra 1. $\mathrm{N}=1065$.

\begin{tabular}{|ll|c|c|c|c|c|c|c|c|}
\hline \multicolumn{1}{|c|}{ Item } & 1 & 2 & 3 & 4 & 5 & 6 & 7 & 8 \\
\hline 1 & Me cuido bien físicamente & & .31 & .52 & .33 & .30 & .36 & .08 & .07 \\
\hline 2 & Realizo tres comidas al día & .31 & & .25 & .57 & .16 & .33 & .11 & .02 \\
\hline 3 & Realizo ejercicio físico durante ... & .52 & .25 & & .28 & .29 & .31 & .02 & -.02 \\
\hline 4 & Tengo horarios fijos para mis comidas & .33 & .57 & .28 & & .24 & .37 & .12 & .07 \\
\hline 5 & Bebo más de 6 vasos de agua al día & .30 & .16 & .29 & .24 & & .41 & .01 & -.03 \\
\hline 6 & Consumo 2 o más piezas de fruta al día & .36 & .33 & .31 & .37 & .41 & & .08 & .08 \\
\hline 7 & Consumo tábaco & .08 & .11 & .02 & .12 & .01 & .08 & & .48 \\
\hline 8 & Consumo bebidas alcohólicas & .07 & .02 & -.02 & .07 & -.03 & .08 & .48 & \\
\hline
\end{tabular}

Tabla 3: Autovalores y porcentaje de varianza explicada por los factores retenidos. Análisis factorial exploratorio Submuestra 1. Solución rotada.

\begin{tabular}{|c|c|c|c|}
\hline Factor & Autovalor & \% varianza & \% acumulado \\
\hline Alimentación & 1.63 & 27.17 & 27.17 \\
\hline Salud Física & 1.52 & 25.38 & 52.55 \\
\hline Hidratación & 1.39 & 23.10 & 75.65 \\
\hline
\end{tabular}

Tabla 4: Ítems agrupados por factor.

Análisis factorial exploratorio Submuestra 1. Solución rotada.

\begin{tabular}{|ll|c|c|c|}
\hline \multirow{2}{*}{ İ́tem } & \multicolumn{2}{c|}{ Factor } \\
\cline { 3 - 5 } & 1 & 2 & 3 \\
\hline 1 & Me cuido bien físicamente & & .81 & \\
\hline 2 & Realizo tres comidas al día & .87 & & \\
\hline $3 \quad$ Realizo ejercicio físico durante ... & & .87 & \\
\hline 4 & Tengo horarios fijos para mis comidas & .83 & & \\
\hline 5 & Bebo más de 6 vasos de agua al día & & & .88 \\
\hline 6 & Consumo 2 o más piezas de fruta al día & & & .71 \\
\hline
\end{tabular}




\section{Análisis factorial exploratorio (segunda solución factorial)}

Con el fin validar la solución factorial obtenida con la submuestra 1 , se realizó nuevamente un análisis de factores principales con los datos de la submuestra 2; encontrándose de nuevo una estructura de tres factores o factores: Alimentación con un $27.54 \%$ de la varianza explicada, Salud Física con un $24.56 \%$ de la varianza e Hidratación con un $23.30 \%$. El conjunto de los factores principales seleccionados explicaron el $75.40 \%$ de la varianza (Tablas 5 y 6 ). La significación del Test de Barlett (1369.881; $\mathrm{p}<$.0001) y el KMO (.754) muestran, además, una adecuada correlación entre los ítems y una buena adecuación muestral respectivamente.

Tabla 5: Autovalores y porcentaje de varianza explicada por los factores retenidos. Análisis factorial exploratorio Submuestra 2. Solución rotada.

\begin{tabular}{|c|c|c|c|}
\hline Factor & Autovalor & \% varianza & \% acumulado \\
\hline 1. Alimentación & 1.65 & 27.54 & 27.54 \\
\hline 2. Salud Física & 1.47 & 24.56 & 52.10 \\
\hline 3. Hidratación & 1.40 & 23.30 & 75.40 \\
\hline
\end{tabular}

Tabla 6: Ítems agrupados por factor.

Análisis factorial exploratorio Submuestra 2. Solución rotada

\begin{tabular}{|ll|c|c|c|}
\hline \multirow{2}{*}{\multicolumn{1}{|c|}{ İtem }} & \multicolumn{2}{c|}{ Factor } \\
\cline { 3 - 5 } & & 1 & 2 & 3 \\
\hline 1 & Me cuido bien físicamente & & .81 & \\
\hline 2 & Realizo tres comidas al día & .85 & & \\
\hline 3 & Realizo ejercicio físico durante ... & & .84 & \\
\hline 4 & Tengo horarios fijos para mis comidas & .85 & & \\
\hline 5 & Bebo más de 6 vasos de agua al día & & & .89 \\
\hline 6 & Consumo 2 o más piezas de fruta al día & & & .71 \\
\hline
\end{tabular}

Congruencia entre los factores de las dos soluciones factoriales (validación cruzada).

Los valores de los coeficientes de congruencia y de los coeficientes de correlación de Pearson entre los pesos factoriales de los factores obtenidos en los análisis factoriales exploratorios llevados a cabo con las submuestras 1 y 2; indican, de acuerdo a Cureton y D'Agostino (1983) una alta congruencia entre pares de factores (Tabla 7 ).

Tabla 7: Coeficientes de congruencia y de correlación de Pearson entre pesos factoriales. Análisis factoriales exploratorios submuestras 1 y 2.

\begin{tabular}{|c|c|c|}
\hline Factor & $\begin{array}{c}\text { Coeficiente de } \\
\text { congruencia }\end{array}$ & $\begin{array}{c}\text { Coeficiente de } \\
\text { correlación }\end{array}$ \\
\hline 1. Alimentación & .999 & .999 \\
\hline 2. Salud Física & .999 & .999 \\
\hline 3. Hidratación & .999 & .999 \\
\hline
\end{tabular}

Fiabilidad de las subescalas (consistencia interna)

De las subescalas (factores) resultantes en los análisis factoriales exploratorios, de ambas submuestras, solo Alimentación posee un alfa superior a .7, evidenciando una consistencia interna adecuada para este tipo de subescalas, particularmente si se considera el número reducido de ítems; mientras que Salud Física e Hidratación presentan baja consistencia interna (Tabla 8). 
Tabla 8: Coeficientes de consistencia interna de los factores obtenidos en los análisis factoriales exploratorios submuestras 1 y 2 .

\begin{tabular}{|c|c|c|}
\hline Factor & a Submuestra 1 & $\alpha$ Submuestra 2 \\
\hline 1. Alimentación & .718 & .727 \\
\hline 2. Salud Física & .635 & .601 \\
\hline 3. Hidratación & .577 & .590 \\
\hline
\end{tabular}

\section{Análisis factorial confirmatorio (primera solución factorial)}

Los resultados de la Tabla 9 del análisis factorial confirmatorio (Submuestra 2) indican que el modelo de medición de tres factores ajusta de manera óptima (GFI .992; RMSR 0.204; RMSEA .054; ECVI 0.053) y de acuerdo a las medidas de ajuste incremental y de parsimonia; salvo por el índice de calidad de ajuste de parsimonia PGFI (Tablas 10 y 11) significativamente superior, a los modelos independiente y de 1 factor, y similar al modelo saturado.

Tabla 9: Medidas absolutas de ajuste para los modelos generados.

Primer análisis factorial confirmatorio submuestra $2 ;{ }^{* * *} \mathrm{p}<.01$.

\begin{tabular}{|l|c|c|c|c|c|}
\hline Modelo & \multicolumn{5}{|c|}{ Índice de Ajuste } \\
\hline & $\chi^{2}$ & GFI & RMSR & RMSEA & ECVI \\
\hline Independiente & $1373.686^{* * *}$ & .620 & 2.402 & .298 & 1.355 \\
\hline Saturado & 0 & 1 & 0 & & 0.041 \\
\hline 1 factor & $235.005^{* * *}$ & .925 & 0.577 & .157 & 0.253 \\
\hline 3 factores & $23.940^{* * *}$ & .992 & 0.204 & .054 & 0.053 \\
\hline
\end{tabular}

Tabla 10: Medidas de ajuste incremental para los modelos generados.

Primer análisis factorial confirmatorio Submuestra 2.

\begin{tabular}{|l|c|c|c|c|}
\hline \multicolumn{1}{|c|}{ Modelo } & \multicolumn{4}{|c|}{ Índice de Ajuste } \\
\hline & AGFI & TLI & NFI & CFI \\
\hline Independiente & .468 & 0 & 0 & 0 \\
\hline Saturado & & & 1 & 1 \\
\hline 1 factor & .826 & .723 & .829 & .834 \\
\hline 3 factores & .972 & .967 & .983 & .987 \\
\hline
\end{tabular}

Tabla 11: Medidas de ajuste de parsimonia para los modelos generados. Primer análisis factorial confirmatorio submuestra 2.

\begin{tabular}{|l|c|c|c|c|}
\hline \multicolumn{1}{|c|}{ Modelo } & \multicolumn{4}{|c|}{ Índice de Ajuste } \\
\hline & PNFI & PGFI & CMIN/GL & AIC \\
\hline Independiente & 0 & .443 & 91.579 & 1385.686 \\
\hline Saturado & 0 & & & 42.000 \\
\hline 1 factor & .497 & .397 & 26.112 & 259.005 \\
\hline 3 factores & .393 & .283 & 3.990 & 53.940 \\
\hline
\end{tabular}

En la figura 1 se presenta el modelo de medición para los 6 ítems agrupados en tres factores, incluyendo los coeficientes de regresión estandarizados entre ítems y factores y las saturaciones factoriales estandarizadas (comunalidades) de cada uno de los ítems. 


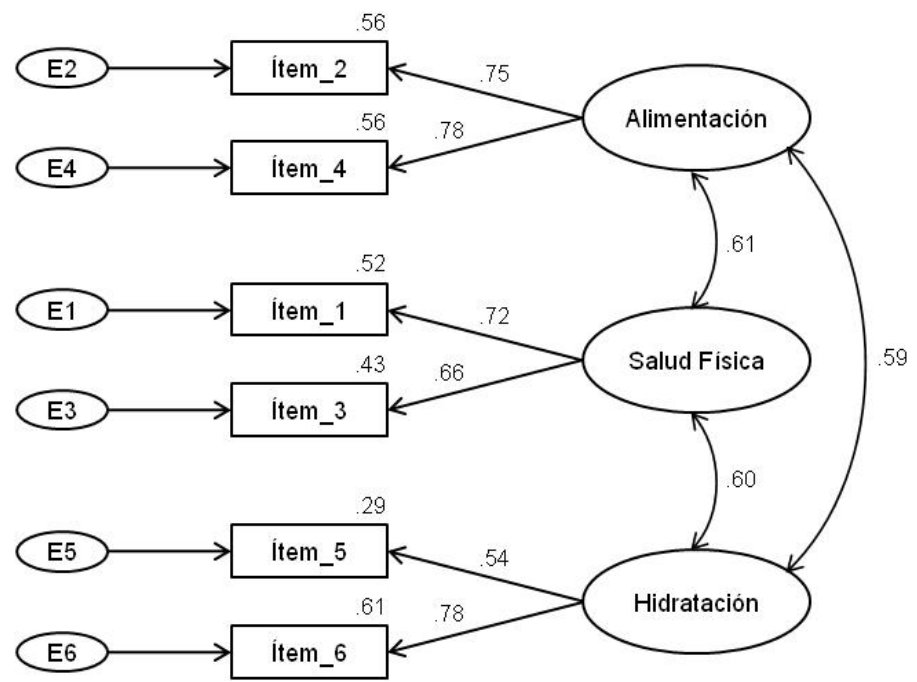

Fig. 1: Modelo de medición para la escala. Análisis factorial confirmatorio submuestra 2.

El primer factor: Alimentación, presenta saturaciones factoriales estandarizadas elevadas (mayores a .50). En el segundo factor, Salud Física, los dos ítems resultan bien explicados a partir del constructo hipotetizado, como muestran una saturación de .52 en el ítem 1 y de .43 en el ítem 3. Finalmente, el tercer factor, Hidratación, presenta una saturación factorial de .29 en el ítem 5 y de .61 en el ítem 6.

Por su parte, la Tabla 12 ofrece la estimación de las correlaciones entre los tres factores de la escala. Todos los factores correlacionan entre sí de forma estadísticamente significativa $(p<.01)$. Las correlaciones entre los factores pueden considerarse altas, con un valor promedio de .600, lo cual muestra que a medida que aumenta la autoeficacia percibida en alguno de los factores, también aumenta en los otros dos.

Tabla 12: Correlaciones entre los tres factores de la escala.

Primer análisis factorial confirmatorio submuestra 2.

\begin{tabular}{|l|c|c|c|}
\hline \multicolumn{1}{|c|}{ Factor } & Alimentación & Salud Física & Hidratación \\
\hline Alimentación & & .609 & .595 \\
\hline Salud Física & .609 & & .597 \\
\hline Hidratación & .595 & .597 & \\
\hline
\end{tabular}

\section{Análisis factorial confirmatorio (segunda solución factorial)}

La estructura factorial analizada plantea un modelo de tres factores, con adscripciones de los ítems basados en análisis factorial confirmatorio de la submuestra 2. De acuerdo a los resultados de la Tabla 13, el análisis factorial confirmatorio de la submuestra 1 indica que el modelo de medición de tres factores es óptimo (GFI .995; RMSR 0.175; RMSEA .040; ECVI 0.044) y de acuerdo a las medidas de ajuste incremental y de parsimonia (Tablas 14 y 15) significativamente superior, al modelo independiente y muy similar al modelo saturado.

Tabla 13: Medidas absolutas de ajuste para los modelos generados.

Segundo análisis factorial confirmatorio submuestra $1 ;{ }^{* * *} p<.01$.

\begin{tabular}{|l|c|c|c|c|c|}
\hline \multicolumn{1}{|c|}{ Modelo } & \multicolumn{5}{c|}{ Índice de Ajuste } \\
\hline & $\chi^{2}$ & GFI & RMSR & RMSEA & ECVI \\
\hline Independiente & $1438.483^{* * *}$ & .621 & 2.47 & .299 & 1.363 \\
\hline Saturado & 0 & 1 & 0 & & 0.039 \\
\hline 3 factores & $16.305^{* * *}$ & .995 & .175 & .040 & 0.044 \\
\hline
\end{tabular}


Tabla 14 Medidas de ajuste incremental para los modelos generados.

Segundo análisis factorial confirmatorio submuestra 1.

\begin{tabular}{|l|c|c|c|c|}
\hline \multicolumn{1}{|c|}{ Modelo } & \multicolumn{4}{|c|}{ Índice de Ajuste } \\
\hline & AGFI & TLI & NFI & CFI \\
\hline Independiente & .444 & 0 & 0 & 0 \\
\hline Saturado & & & 1 & 1 \\
\hline 3 factores & .982 & .982 & .989 & .993 \\
\hline
\end{tabular}

Tabla 15: Medidas de ajuste de parsimonia para los modelos generados. Segundo análisis factorial confirmatorio submuestra 1.

\begin{tabular}{|l|c|c|c|c|}
\hline \multicolumn{1}{|c|}{ Modelo } & \multicolumn{4}{|c|}{ Índice de Ajuste } \\
\hline & PNFI & PGFI & CMIN/GL & AIC \\
\hline Independiente & 0 & .444 & 95.899 & 1450.483 \\
\hline Saturado & 0 & & & 42.000 \\
\hline 3 factores & .395 & .284 & 2.717 & 46.305 \\
\hline
\end{tabular}

En la figura 2 se presenta el modelo de medición para los 6 ítems agrupados en tres factores, incluyendo los coeficientes de regresión estandarizados entre ítems y factores y las saturaciones factoriales estandarizadas (comunalidades) de cada uno de los ítems.

El primer factor: Alimentación, presenta saturaciones factoriales estandarizadas elevadas (mayores a .49). En el segundo factor, Salud Física, los dos ítems resultan bien explicados a partir del constructo hipotetizado, como muestran una saturación de .60 en el ítem 1 y de .45 en el ítem 3. Finalmente, el tercer factor, Hidratación, presenta una saturación factorial de .30 en el ítem 5 y de .55 en el ítem 6.

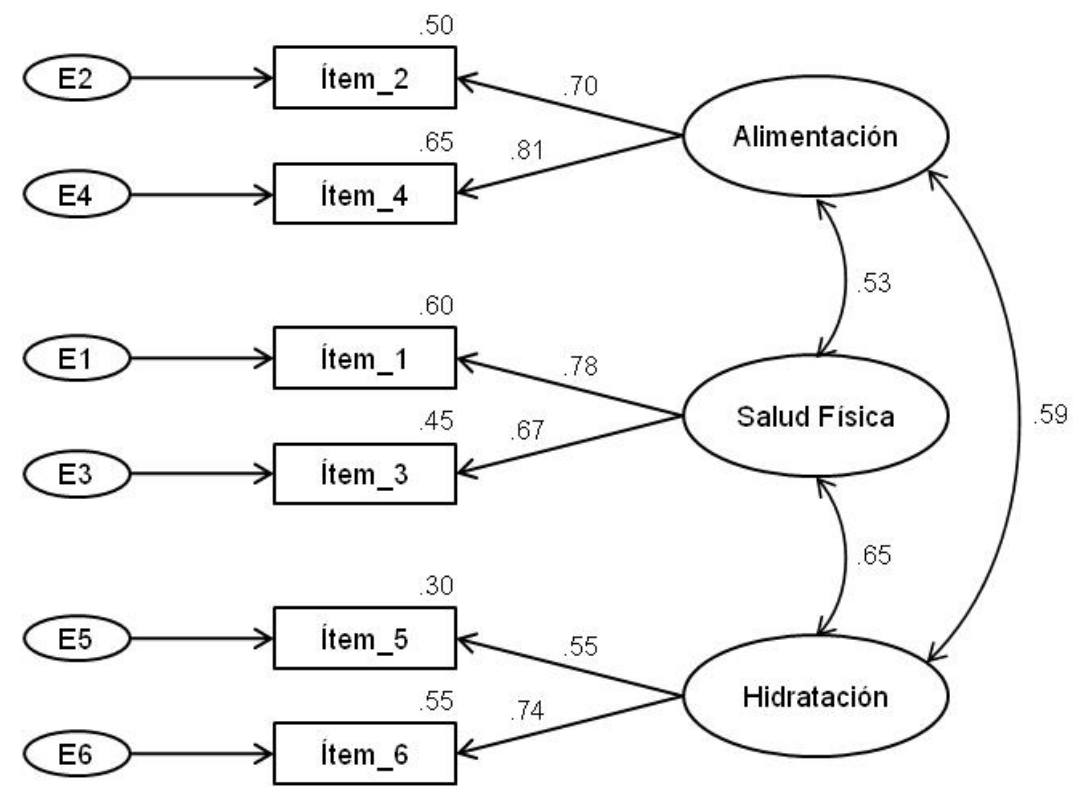

Fig. 2: Modelo de medición para la escala. Análisis factorial confirmatorio submuestra 1.

Por su parte, la Tabla 16 ofrece la estimación de las correlaciones entre los tres factores de la escala. Todos los factores correlacionan entre sí de forma estadísticamente significativa $(p<.01)$. Las correlaciones entre los factores pueden considerarse altas, con un valor promedio de .589 , lo cual muestra que a medida que aumenta la autoeficacia percibida en alguno de los factores, también aumenta en los otros dos. 
Tabla 16: Correlaciones entre los tres factores de la escala.

Segundo análisis factorial confirmatorio submuestra 1.

\begin{tabular}{|l|c|c|c|}
\hline \multicolumn{1}{|c|}{ Factor } & Alimentación & Salud Física & Hidratación \\
\hline Alimentación & & .535 & .588 \\
\hline Salud Física & .535 & & .651 \\
\hline Hidratación & .588 & .651 & \\
\hline
\end{tabular}

Congruencia entre los factores de las dos soluciones factoriales (validación cruzada)

Los valores de los Coeficientes de Congruencia y de los Coeficientes de correlación de Pearson entre los pesos factoriales (coeficientes de regresión estandarizados) de los factores obtenidos en los análisis factoriales confirmatorios llevados a cabo con las submuestras 1 y 2; indican, de acuerdo a lo sugerido por Cureton y D'Agostino (1983) una alta congruencia entre pares de factores. Lo que significa que los resultados del modelo son plenamente confirmatorios (Tabla 17).

Tabla 17. Coeficientes de Congruencia y de correlación de Pearson entre pesos factoriales. Análisis factoriales confirmatorios submuestras 1 y 2 .

\begin{tabular}{|l|c|c|}
\hline \multicolumn{1}{|c|}{ Factor } & $\begin{array}{c}\text { Coeficiente de } \\
\text { Congruencia }\end{array}$ & $\begin{array}{c}\text { Coeficiente de } \\
\text { correlación }\end{array}$ \\
\hline Alimentación & .994 & .999 \\
\hline Salud Física & .998 & .999 \\
\hline Hidratación & .997 & .999 \\
\hline
\end{tabular}

Fiabilidad de las subescalas

Las subescalas (constructos) resultantes en los análisis factoriales confirmatorios, de ambas submuestras, poseen fiabilidades inferiores a .7, evidenciando, de acuerdo a Hair et al. (1999) una fiabilidad inadecuada, probablemente debido al número reducido de ítems. En cuanto a la varianza extraída ninguno de los factores (constructos) logra el mínimo sugerido (Tabla 18).

Tabla 18: Fiabilidad y varianza extraída de los constructos obtenidos en los análisis factoriales confirmatorios submuestras 1 y 2.

\begin{tabular}{|c|c|c|c|c|c|}
\hline Constructo & \multicolumn{2}{|c|}{ Submuestra 1 } & & \multicolumn{2}{c|}{ Submuestra 2 } \\
\hline & Fiabilidad & Varianza & & Fiabilidad & Varianza \\
\hline Alimentación & .507 & .340 & & .499 & .336 \\
\hline Salud Física & .371 & .229 & & .437 & .283 \\
\hline Hidratación & .343 & .227 & & .309 & .195 \\
\hline
\end{tabular}

\section{DISCUSIÓN FINAL}

En cuanto al Análisis Factorial Exploratorio llevado a cabo para determinar el número mínimo de factores comunes capaces de reproducir, de un modo satisfactorio, las correlaciones observadas entre los 6 ítems del instrumento autoeficacia en conductas de cuidado de la salud física (sin considerar los ítems consumo de tabaco y consumo de alcohol), reveló una estructura de tres factores: Alimentación, Salud Física e Hidratación. Los factores de ambas submuestras evidenciaron una consistencia interna adecuada para Alimentación y baja para Salud Física e Hidratación (muy probablemente debido al número reducido de ítems en cada uno de ellos); así 
como una alta congruencia entre pares de factores, particularmente si se considera el número reducido de ítems en cada uno de ellos. Por otra parte, el Análisis Factorial Confirmatorio indicó que el modelo de medición de 6 ítems en tres factores ajusta de manera óptima al modelo teórico. Al mismo tiempo que los tres factores así obtenidos presentan en general saturaciones factoriales estandarizadas moderadas. Por su parte los tres factores correlacionan entre sí de forma positiva y estadísticamente significativa lo cual muestra que a medida que aumenta la autoeficacia percibida en alguno de los factores, también aumenta en los otros dos. Conjuntamente con todo lo antes dicho, los valores de los coeficientes de congruencia y de los coeficientes de correlación de Pearson entre los pesos factoriales (coeficientes de regresión estandarizados) de los factores obtenidos en los análisis factoriales confirmatorios llevados a cabo con las submuestras 1 y 2; indican una alta congruencia entre pares de factores. Lo que significa que los resultados del modelo son plenamente confirmatorios.

Por último, en las escalas de autoeficacia habitualmente se les pregunta a las personas acerca de sus capacidades operativas en el presente, no acerca de sus capacidades potenciales o sobre sus capacidades futuras esperadas (Bandura, 2001) mientras que, en el diseño del instrumento empleado en nuestra investigación para medir autoeficacia, además de que el sujeto responde sobre la frecuencia con que actualmente realiza una determinada conducta o se percibe con determinada característica; responde también en forma ideal y si se esfuerza en cambiar, con que frecuencia realizaría o manifestaría dichas acciones y/o habilidades. Esto es, cada sujeto responde a los ítems del instrumento en tres escenarios distintos: Escenario actual, respondiendo en el contexto: actualmente con que frecuencia realiza determinada conducta o se percibe con determinada característica. Escenario ideal, respondiendo en el contexto: con que frecuencia desearía realizar determinada conducta o percibirse con determinada característica. Escenario de cambio, respondiendo en el contexto: si me esfuerzo en cambiar con que frecuencia realizaría determinada conducta o me percibiría con determinada característica.

Para luego a partir de sus respuestas obtener 7 índices: 1) Autoeficacia percibida actualmente.obtenida a partir de las respuestas al escenario actual. 2) Autoeficacia deseada.- obtenida a partir de las respuestas al escenario ideal. 3) Autoeficacia alcanzable en el futuro.- obtenida a partir de las respuestas al escenario de cambio. 4) Grado de insatisfacción o disonancia en la autoeficacia percibida.- obtenida a través de la diferencia entre el índice 2 y 1 (ideal menos actual). 5) Posibilidad de mejoría en la autoeficacia percibida.- obtenida a través de la diferencia entre el índice 3 y 1 (cambio menos actual). 6) Proporción de autoeficacia percibida en relación a la deseada.- obtenida a partir del índice 1 y 2 (actual entre ideal por 100) y 7) Proporción de autoeficacia percibida en relación a la alcanzable.- obtenida a partir del índice 1 y 3 (actual entre cambio por 100).

Esto permite, entre otras cosas, generar disonancia cognoscitiva en el sujeto en relación su autoeficacia percibida en el presente, el nivel de autoeficacia deseado o ideal, y la posibilidad de mejoría en la autoeficacia percibida. La disonancia cognoscitiva hace referencia a la tensión o desarmonía interna del sistema de ideas, creencias, emociones y actitudes (cogniciones) que percibe una persona al mantener al mismo tiempo dos pensamientos que están en conflicto, o por un comportamiento que entra en conflicto con sus creencias (Festinger, 1957). En cuanto hace su aparición la disonancia brota una fuerza igual y de signo contrario para reducirla; en otras palabras, la disonancia actúa del mismo modo que un impulso, necesidad o estado de tensión. La presencia de la disonancia lleva a una acción para reducirla de idéntica forma que, por ejemplo, el hambre lleva a una acción para evitarla.

\section{CONCLUSIONES}

De los resultados mostrados, de su análisis y de su discusión, se pueden obtener las siguientes conclusiones, sobre las propiedades psicométricas de la Escala Autoeficacia en Conductas de Cuidado de la Salud Física: 1) el análisis de las propiedades psicométricas, ha mostrado que una estructura trifactorial es viable y adecuada de acuerdo a los requisitos psicométricos establecidos cuando los informantes son los propios alumnos; 2) La estructura de tres factores, atendiendo a criterios estadísticos y sustantivos, ha mostrado adecuados indicadores de ajuste, de fiabilidad 
(salvo en los factores Salud Física e Hidratación) y de validez; 3) como la validez factorial de los instrumentos de medida debe ser demostrada con muestras que presenten diferencias tanto poblacionales como culturales, consideramos que más estudios son necesarios con el fin de corroborar o refutar los datos obtenidos en la presente investigación.

\section{REFERENCIAS}

Arbuckle, J., AMOS users guide version 16.0 (2007).

Bandura, A., Exercise of personal agency throught the self-efficacy mechanism. In R. Schwarzer (Ed.), Self-efficacy: thought control of action, pp. 3-38, Hemisphere, Washington, DC, (1992).

Bandura, A., Self-efficacy: The exercise of Control, Freeman, New York (1997).

Bandura, A., Guía para la construcción de escalas de autoeficacia (2001). http://www.revistaevaluar.com.ar/effguideSpanish.htm. Acceso: 16 mayo (2011)

Batista, J. M. y Coenders, G., Modelos de ecuaciones estructurales, Cuadernos de estadística 6. 59-102, La Muralla, Madrid (2000).

Blanco, H. y otros siete autores, Editor para la construcción y aplicación de escalas por medio de una PC. Tecnociencia Chihuahua, 1(1), 55-59 (2007).

Brown, S. D., Tramayne, S., Hoxha, D., Telander, K., Fan, X. y Lent, R. W., Social cognitive predictors of college students'academic performance and persistence: a meta-analytic path analysis, Journal of Vocational Behavior, ISSN: 0001-8791 (en línea), 72 (3), 298-308, 2008. http://dx.doi.org/10.1016/j.jvb.2007.09.003. Acceso: 3 de agosto (2011).

Campos, S. y Pérez, J., Autoeficacia y conflicto decisional frente a la disminución del peso corporal en mujeres. Revista Chilena de Nutrición, ISSN: 0717-7518 (en línea), 34(3), 1-17, 2007. http://www.scielo.cl/scielo.php?pid=S0717-75182007000300004\&script=sci_arttext. Acceso: 3 de agosto (2011).

Choi, N., Sex role group differences in specific, academic, and general self-efficacy. The Journal of Psychology, 138, 149-159 (2004).

Costello, A. y Osborne, J., Best practices in exploratory factor analysis: four recommendations for getting the most from your analysis. Practical Assessment, Research and Evaluation, ISSN: 15317714, 10(7), 1-9, 2005. http://pareonline.net/pdf/v10n7.pdf. Acceso: 9 de Mayo (2011)

Cureton, E. E. y D'Agostino, R. B., Factor Analysis: An applied approach. Lawrence Erlbaum Associates, London (1983).

Elosua, P. y Zumbo, B., Coeficientes de fiabilidad para escalas de respuesta categórica ordenadas, Psicothema, ISSN: 1886-144X (en línea), 20(4), 896-901, 2008. http://www.psicothema.com/pdf/3572.pdf. Acceso: 3 de agosto (2011).

Festinger, L., A theory of cognitive dissonance. Stanford University Press, Stanford, CA (1957).

Gutiérrez, M., Escartí, A. y Pascual, C., Relaciones entre empatía, conducta prosocial, agresividad, autoeficacia y responsabilidad personal y social de los escolares, Psicothema, ISSN: 1886-144X (en línea), 23(1), 13-19, 2011. www.psicothema.com/pdf/3843.pdf. Acceso: 3 de agosto (2011).

Hair, J. F., Anderson, R. E., Tatham R. L. y Black, W. C., Análisis multivariante de datos. Prentice Hall, México (1999). 
Klein-Hessling, J., Lohaus, A. y Ball, J., Psychological predictors of health-related behaviour in children, Psychology, Health y Medicine, 10 (1) 31-43 (2005).

Luszczynska, A., Gibbons, F., Piko, B. y Tekozel, M., Self-regulatory cognitions, social comparison, perceived peers' behaviors as predictors of nutrition and physical activity: $A$ comparison among adolescents in Hungary, Poland, Turkey, and USA. Psychology and Health, 19, 577-593 (2004).

Luszczynska, A., Scholz, U. y Schwarzer, R., The general self-efficacy scale: Multicultural validation studies, The Journal of Psychology, ISSN: 0022-3980, 139 (5), 439-457, 2005. http://userpage.fu-berlin.de/ health/self/gse-multicult_2005.pdf. Acceso: 4 de agosto (2011).

Muñiz, J. y Fonseca-Pedrero, E., Construcción de instrumentos de medida para la evaluación universitaria, Revista de Investigación en Educación, ISSN: 1697-5200 (en línea), 5, 13-25, 2008. http://webs.uvigo.es/reined/ojs/index.php/reined/article/viewFile/40/35. Acceso: 3 de agosto (2011).

Nunnally, J. C., Teoría Psicométrica, 247-281, Trillas, México, (1991).

Olivari, C. y Urra, E., Autoeficacia y conductas de salud, Cienc. enferm., ISSN: 0717-9553 (en línea), 13(1), 9-15 2007. http://www.scielo.cl/pdf/cienf/v13n1/art02.pdf. Acceso: 5 de agosto (2011).

Pajares, F., y Schunk, D. H., Self-Beliefs and School Success: Self-Efficacy, Self-Concept, and School Achievement. In R. Riding y S. Rayner (Eds.), Perception, pp. 239-266 Ablex Publishing, London (2001).

Pérez, E., Cupani, M. y Ayllón, S., Predictores de rendimiento académico en la escuela media: aptitudes, autoeficacia y rasgos de personalidad, Avaliaçao Psicológica, ISSN: 1677-0471 (en línea), 4(1), 1-11, 2005. http://pepsic.bvsalud.org/pdf/avp/v4n1/v4n1a02.pdf. Acceso: 3 de agosto (2011).

Pérez, E., Lescano, C., Zalazar, P., Furlám, L. y Martínez, M., Desarrollo y análisis psicométricos de un Inventario de Autoeficacia para Inteligencias Múltiples en Niños Argentinos. Psicoperspectivas, ISSN: 0718-6924 (en línea), 10 (1), 169-189, 2011. http://www.psicoperspectivas.cl/index.php/psicoperspectivas/article/viewFile/141/129. Acceso: 3 de agosto (2011).

Raviolo, A., Ramirez, P., López, E. A. y Aguilar, A., Concepciones sobre el Conocimiento y los Modelos Científicos: Un Estudio Preliminar, Form. Univ., ISSN: 0718-5006 (en línea), 3(5), 29-36, 2010. http://www.scielo.cl/pdf/formuniv/v3n5/art05.pdf. Acceso: 3 de agosto (2011).

Sniehotta, F., Scholz, U. y Schwarzer, R., Bridging the intention-behaviour gap: planning, selfefficacy, and action control in the adoption and maintenance of physical exercise, Psychology of Health, 20 (2), 143-160 (2005).

Thompson, B., Exploratory and Confirmatory Factor Analysis. Understanding concepts and applications, American Psychological Association, Washington, D C: (2004).

Valiante, G., Writing Self-efficacy and gender orientation: A developmental perspective, a dissertation proposal. Emory University, Atlanta (2000)

Zimmerman, B. J., Self-efficacy and educational development. In A. Bandura (Ed.). Self-efficacy in changing societies, pp. 202-231, Cambridge University Press, New York (1995). 